López ME. y cols.

Rev. Chil. Pediatr. 66 (4); 204-208, 1995

\title{
Hidrato de cloral y midazolam en sedación para electroencefalograma en niños de 1 a 5 años
}

\author{
María Eugenia López B. '; Isabel López S. '; Ledia Troncoso A. ; \\ María de los Angeles Avaria B. '; Fernando Novoa S. ${ }^{2}$
}

\section{Chloral bydrate or midazolam to induce sleep for electroencephalographic records}

The efficacy of chloral hycrate and midazolam os sleep incuctors for eleclroencephalographic recording [EEG] was compared in 59 children who received either chloral hydrate $50 \pm \mathrm{mg} \pm \mathrm{kg} \mid \mathrm{n}: 32$ ) or midazolam $1 \mathrm{mg} \pm \mathrm{kg}$ (n: 27 | by rectal administration. Thirty thrse children who achieved spontaneus sleep were included os a reference group. All children in the chlaral hydrale group satl asleep as compared to $66.6 \%$ in the midazolam group $\{p<0.01$ ). The lelency pe"lod lasted $21.8 \pm 17.5 \mathrm{~min}$ in the chloral hydrate group and $117.5 \pm 47.2 \mathrm{mir}$ in the midazolam group $\left(p<0.0^{*}\right)$. Other varables such os age, sex, psychomolor development or time of cwakening or teeding showed no significant influence on these results. Cur results support the Jse of chloral hydrale by reclal adminisleation in childsen who require sedation for $\mathrm{EEG}$.

[Key words: electroencephalogrophy, sleep induction, shlo:al hydrote, midczolam.]

El adecuado registro electroencefalográfico requiere con cierta frecuencia, en particular si se trata de niños, sedar al paciente. La sedación y la consecuente inducción de sueño, que en algunas oportunidades no se obtiene espontáneamente, disminuyen los artefactos de movimiento que dificultan la interpretación del trazado. Por otra parte los registros en estado de somnolencia y sueño, además de vigitia, permiten detectar anormalidades epilépticas específicas y evaluar el patrón madurativo del paciente.

La decisión de administrar un sedante depende más de la capacidad de cooperación del pacienle que de su edad'. El medicamento sedante que se seleccione debe ser de administración fácil e indolora, efecto rápido, consistente, de duración controlable, sin efectos colaterales o depresión residual ${ }^{2}$. Para el electrocncefalograma (EEG) se requiere específicamente que, además, no altcre los patrones electrofisiologicos normales.

1. Servicio de Neuropsiquiatría Infantil, Hospital Clínico San Borja-Arriarán.

2. Departamento de Pediatría y Cirugía Infantil, Facultad de Medicina Central. Universidad de Chile.
El hidrato de cloral es el sedante más usado para procedimientos indoloros en niños. ${ }^{3}$. Es un tricloroacetaldehído que se metaboliza en el hígado a tricloroetanol, responsable de la acción hipnótica. La concentración plasmática máxima de tricloroetanol ocurre entre 30 y 60 min después de la ingestión ora $]^{4}$. También puede administrarse por vía rectal, con lo que se evita el rechazo debido al mal sabor del medicamento, especialmente en niños pequeños. Por su liposolubilidad se distribuye ampliamente en los tejidos, se conjuga en el hígado con ácido glucurbnico y se excreta por vía renal. Su vida media es de 4 a 12 horas $^{5}$. En dosis mayores a $100 \mathrm{mg}$ * kg puede producir depresión respiratoria, hipotensión arterial y miosis (similar a una intoxicación barbitúrica). Está contraindicado en insuficiencia respiratoria o cardíaca grave, y en falla renal o hepática. En estudios de sedación para electroencefalograma (EEG), tomografía axial computadorizada (TAC) o registro de potenciales evocados se ha usado hidrato de cloral por vía oral, logrando buen éxito en alto porcentaje de casos con dosis entre 25 y $75 \mathrm{mg} \cdot \mathbf{k g}$, lo que da un amplio margen de seguridad $2,6,7$. Como factores negativos se aluden un tiempo de inducción largo e impredecible y efecto prolongado'. 
El midazolam es una benzodiazepina hipnótica de acción ultracorta $(1,5$ a 2,5 h) con propiedades ansiolíticas, amnésticas, anticonvulsivantes y de relajacion muscular. Su efecto hipnótico se debe a que su receptor y el receptor del ácido gamaaminobutírico (GABA) están acoplados a un canal de cloro común y a disminución de la recaptación de GABA ${ }^{8}$. Su anillo imidazol, distinto al de otras benzodiazepinas, da cuenta de su hidrosolubilidad a pH ácido y su liposolubilidad a $\mathrm{pH}$ corporal ${ }^{8}$. Esto hace rápido el equilibrio entre sangre y cerebro, permitiendo titular la dosis durante la infusión endovenosa ${ }^{9}$. En el hígado se metaboliza rápida y completamente a hidroximidazolam, inactivo. Se excreta por el riñón y el hígado. En procedimientos invasivos se usa por vía parenteral, lográndose inconsciencia en pocos minutos con dosis de 0,1 a $0,4 \mathrm{mg} \cdot \mathrm{kg}$, de lo que el paciente se recupera rápidamente después de 15 min, alcanzándose alerta completa en $60 \mathrm{~min}^{10-12}$. Puesto que no irrita las mucosas, la solucion parenteral se puede usar en forma enteral. Por vía oral alcanza una concentración plasmática máxima en una hora, 1legando a la circulación 40 a $50 \%$ de la dosis, debido a extracción hepática de primer paso ${ }^{8}$. En premedicación anestésica se ba obtenido ansjolisis y amnesia en 80 a $90 \%$ de los niños con $0,75 \mathrm{mg} \cdot \mathbf{k g}^{13}$, pero la frecuencia de buen éxito es inferior en niños menores de cinco años, en los cuales puede producir efecto paradojal ${ }^{13,}{ }^{14}$. Por vía rectal se obtiene concentración plasmática máxima en 16 a 20 minutos, lo que coincide con un efecto ansiolítico a pesar de alcanzar concentraciones de $100 \mathrm{ng} / \mathrm{ml}$ en la sangre, consideradas hipnóticas. Las dosis usadas oscilan entre 0,3 y $5 \mathrm{mg}$ - kg. Sobre $1 \mathrm{mg} \cdot \mathrm{kg}$ todos los pacientes se han sedado en forma adecuada. Sobre $2 \mathrm{mg} \cdot \mathbf{k g}$ existe una demora en la recuperación mayor a 60 minutos. No se han observado cambios en los parámetros hemodinámicos ni respiratorios con estas dosis. La administración de midazolam induce ritmos rápidos y desaparición de ondas vértex y husos de sueño en los registros electroencefalográgicos ${ }^{15-17}$.

Se ha descartado el uso de los barbitúricos de administración enteral, pentobarbital y secobarbital, por su efecto impredecible (debido a absorción irregular), vida media larga y no estar disponibles en Chile ${ }^{1,2,5}$. Tampoco se emplean, por no estar probada su inocuidad en niños y descritas las dosis según peso corporal, las benzodiazepinas hipnóticas de acción corta -como el brotizolam y triazolam- y la zopiclona, una ciclopirrolona con perfil farmacológico semejante.

El propósito de este estudio fue comparar la eficacia del hidrato de cloral y de midazolam -administrados por vía rectal- en la rapidez para inducir sueño, calidad y duración de éste y eventuales cambios en el trazado electroencefalográfico atribuibles a estos medicamentos, en niños de edad preescolar que requieran EEG.

\section{Pacientes y Método}

Se estudiaron 92 niños de 1 a 5 ahos, enviados a nuestra unidad para registro EEG entre junio y diciembre del año 1993, excluyendo tos que estuvieran en tratamiento con barbitúricos o benzodiazepinas, ya que estos medicamentos modifican el trazado del EEG

Se pidió a jas madres que en el día de registro despertaran a sus hijos a las 6 a.m. y se les citó de preferencia durante la manana. A los niffos que no estaban somnolientos o dormidos en el momento de iniciar el examen ni presentaban contraindicaciones para la sedación, se les administro $50 \mathrm{mg} \cdot \mathrm{kg}$ de bidrato de cloral en solución al $5 \%$ o midazolam en solución parenteral de $5 \mathrm{mg} / \mathrm{ml}$, en dosis de $1 \mathrm{mg} \cdot \mathrm{kg}$, ambos por vía rectal. Debida al pequefto volumen de inidazolam, éste fue impulsado con $3 \mathrm{ml}$ de solución de $\mathrm{NaCl} 0,9 \%$, para evilar que quedara en la sonda. La asiguación del sedante se realizó en cada nif́o lanzando una moneda al aire: el lado cara correspondía a hidrato de cloral y el sello a midazolam. Para el caso de que ocurriese depresión respiratoria estaba previsto el empleo endovenoso de flumazemil en el esquema habitual ${ }^{18,19}$. Como grupo de referencia se emplearon los niños que durmieron espontáneamente

Se incluyeron en total 92 niños (63\% varones) que se distribuyeron en los grupos de estudio hidrato de cloral ( $n=32$; varones $22 ; 7$ niños con retraso psicomotor: edad $27,9 \pm 11,3 \mathrm{~m}$ ) y midazolam ( $\mathrm{n}=27$; varones $16 ; 5$ niños con retraso psicomotor; edad $30,1 \pm 14,2 \mathrm{~m}$ ) y controles 0 de referencia $(a=33$; varones $20 ; 6$ riños con retraso psicomotor; edad 34,2 $\pm 14,2 \mathrm{~m}$ ), que no mostraron diferencias significativas en sexo, edad o desarrollo psicomotor y cuyos diagnosticos clínicos se resumen en la tabla 1

Se consignó la hora del último despertar y última aljmentación, hora de sedación, hora de suefio, calidad del sueño evaluada según cantidad de movimientos y despertares durante el procedimiento, hora del despertar despues del exarnen (espontáneo o inducido) y efectos colaterales durante el periodo de observación.

Los trazados EEG fueron analizados por dos médicos electroencefalografistas (EM, LT) que desconocían si se habla administrado sedante, a quienes se solicitó opinar si encontraban o no alteraciones del patrón de base atribuibles a un sedaule (impregnación), y a qué medicamento las atribuian. 


\section{Tabla}

Diagnósticos de referencia a electroencefalograma de los pacientes premedicados con hidrato de cloral, midazolam y controles no medicados

\begin{tabular}{lccc}
\hline & $\begin{array}{c}\text { H de } \\
\text { clorad }\end{array}$ & $\begin{array}{c}\text { Mida- } \\
\text { zolam }\end{array}$ & $\begin{array}{c}\text { Con- } \\
\text { troles }\end{array}$ \\
\hline $\begin{array}{l}\text { Epilepsia o crisis no } \\
\text { provocadas }\end{array}$ & 8 & 7 & 6 \\
$\begin{array}{l}\text { Crisis no epilépticas } \\
\text { Convulsiones febriles } \\
\text { Trastornos del desarrollo }\end{array}$ & 14 & 0 & 7 \\
$\begin{array}{l}\text { y conductuales } \\
\text { Cefalea }\end{array}$ & 1 & 10 & 9 \\
Trastomos del sueño & 1 & 1 & 4 \\
Otros & 0 & 2 & 0 \\
\hline Total & 5 & 5 & 6 \\
\hline
\end{tabular}

Se registro la frecuencia de diagnósticos de impregласіón medicamentosa, en los tres grupos de pacientes, la frecuencia de identificación correcta del sedarte utilizado y los elementos del trazado que orientaron a este diagnóstico.

Se analizaron los grupos de estudio en relación a las variables dependientes (latencia de sueño después de la sedación, calidad y đuración de éste) y la influencia sobre ellas de las probables variables intervinientes (sexo. edad. presencia de retraso del desarrollo psicomotor, período de vigilia previo e intervalo entre la alimentacion y la se. dación). Las diferencias de frecuencia se estudiaron con análisis de Chi cuadrado. Las diferencias de promedio se estudiaron con prueba $t$ de Student para muestras independientes.

\section{Resultados}

En todos los niños en que se empleó hidrato de cloral se logró el sueño, con una latencia promedio de $21,8 \pm 17,5 \mathrm{~min}$, variando entre $3 \mathrm{y}$ 90 min, siendo menor a 15 min en $56,3 \%$ y menor a $30 \mathrm{~min}$ en $84,3 \%$ de los casos. Esto difiri6 significativamente de 10 sucedido con midazolam en que solo $18(66,6 \%)$ de los niños conciliaron el sueño $(p<0,01)$, con latencia promedio de $117,5 \pm 47,2 \mathrm{~min}(\mathrm{p}<0,01)$ y sólo en tres casos antes de transcurridos $60 \mathrm{~min}$. El tiempo de latencia hasta la inducción de sueño no varió con el sexo o la edad y fue algo mayor en los niños con retraso psicomotor que ricibieron midazolam, pero en este caso no se aplić́ análisis estadístico por el bajo número de pacientes. Tampoco influyeron el tiempo de vigi- lia previo a la sedación ni el intervalo entre alimentación y sedación.

La duración del sueño fue $61 \pm 31,2$ min en los pacientes que recibieron hidrato de cloral; $45,9 \pm 12,2 \mathrm{~min}$ en los tratados con midazolam y $35,1 \pm 18,4 \mathrm{~min}$ en los niños no tratados, siendo significativamente mayor con hidrato de cloral que con midazolam $(\mathrm{p}<0,05)$ y controles $(p<0,01)$ y con midazolam que en controles $(p<0,05)$. La calidad del sueño fue tranquila en $68,8 \%$ de los niños que recibieron hidrato de cloral, pero sólo en $33,3 \%$ y $24,4 \%$, respectivamente, de los niños tratados con midazolam o sin sedantes $(\mathrm{p}<0,01)$. No se observaron efectos secundarios clínicos en ninguno de los niños que recibieron medicamentos.

Ś́lo se obtuvieron 21 trazados bajo influencia đe midazolam, pues en los seis niños restantes el registro no fue posible por no haber logrado calmarles. Los electroencefalografistas opinaron que habia signos de impregnación medicamentosa en $19(59,4 \%)$ de los 32 trazados de los niños tratados con hidrato de cloral, en $10(47,6 \%)$ de los 21 bajo acción de midazolam $y$ en $8(24,2 \%)$ de los 33 EEG del grupo control, lo que se atribuyb en 5 de 8 casos a hidrata de cloral. La frecuencia con que se describieron signos de impregnación resultó significativamente mayor para los grupos sedados (chi: 8,46 , $p<0,05$ ), principalmente por la diferencia entre los grupos hidrato de cloral y controles (chi: $6,88, \mathrm{p}<0,01)$. La identificación correcta del medicamento se dio en $13(68,6 \%)$ de los trazados considerados impregnados para los casos en que este era hidrato de cloral y $2(20 \%)$ cuando se trataba de midazolam ( $<<0,05$ ). El elemento que más orientó a sospechar que se había empleado algún sedante fue la sobrecarga de ritmos rápidos, registrados en $15 / 19$ trazados calificados como impregnados en los niños que recibieron hidrato de cloral, 6/10 EEG "impregnados" entre los casos medicados con midazolam y en $6 / 8$ controles con trazados declarados bajo efecto medicamentoso. Este elemento fue adjudicado por los electroencefalografistas al uso de hidrato de cloral o a sedante no especificado. El segundo elemento "orientador", que no fue atribuido de preferencia a ningún medicamento, fue el inicio de sueño en etapas ПI y III no REM, que se observ6́ asociado a la taquiarritmia en seis trazados del grupo con hidrato de cloral, cuatro controles y uno con midazolam. 


\section{Comentario}

En este trabajo se confirma la efectividad del hidrato de cloral como sedante para procedimientos indoloros en niños, superando el éxito alcanzado en trabajos previamente publicados, que era cercano al $85 \%^{1,2,6, ?}$. No tuvimos fracaso en la sedacion como se ha observado en niños en edad preescolar con este medicamen$t^{7}{ }^{14}$. Tampoco hubo falla ni demora en la inducción de sueño en niños con retraso del desartollo, como se ha descrito ${ }^{6,7}$, aunque dado el tamaño de la muestra no es posible sacar conclusiones definitivas a este respecto. La Iatencia de sueño fue bastante menor a lo encontrado en otros estudios que utilizaron dosis similares o mayores, en los cuales era alrededor de 60 $\min ^{1,2}$. El mayor porcentaje de éxito y el menor tiempo de inducción observado en nuestro estudio podría estar dado por el uso de la vía rectal, que permite la llegada del medicamento al lugar de absorción en menor tiempo que la vía oral, lo que también se ha observado con otros fármacos, como las benzodiazepinas. La duración del efecto hipnotico fue larga, lo que sí coincide con otras observaciones ${ }^{1}$. Esto puede considerarse una desventaja, aunque permite la obtención de trazados prolongados si se requiere. La calidad del sueño fue satisfactoria en la mayoría de los casos, disminuyendo el riesgo de artefactos de movimiento en el trazado. Se considera que los efectos del hidrato de cloral sobre el trazado electroencefalográfico no son relevantes. Aunque en este estudio se observaron en un porcentaje significativo de los trazados, éstos no afectaron en forma importante su evaluación.

Con midazolam no se cumplieron los requisitos antes mencionados para una buena sedación, por el alto porcentaje de fracasos y la larga latencia del sueño inducido. Aún más, no es posible desechar la posibilidad que el sueño no se debiese realmente a efecto medicamentoso, ya que las concentraciones plasmáticas y efectos clínicos máximos aparecen en menos de $30 \mathrm{mi}$ nutos cuando el medicamento se suministra por vía rectal ${ }^{15}$. Los signos interpretados como impregnación medicamentosa en los trazados no descartan esta posibilidad, ya que en un estudio del efecto del midazolam endovenoso sobre el EEG, los ritmos rápidos persistieron hasta 60 min đespués de recuperada la conciencia ${ }^{17}$. En este grupo no se apreciaron las alteraciones en las etapas de sueño descritas en esa misma publicación. Cuando se ha usado el midazolam. para premedicación anestésica en dosis similares a las usadas en este trabajo, no se ha logrado inducir sueño, pero se ha producido un efecto calmante que no fue observado por nosotros. Esto puede deberse a un efecto paradojal, que ha sido descrito con benzodiazepinas a esta edad $^{13}$.

Aunque en los niños que no recibieron medicamento el sueño fue breve y su calidad no fue optima, se logró un trazado útil en todos ellos. La interpretación de signos de efectos medicamentosos en algo menos de la cuarta parte de ellos debe imponer una nota de cautela al significado de este tipo de observaciones.

En la decisión de sedar a un niño se deben considerar también algunas precauciones y contraindicaciones de esta acción, como antecedentes de alergia a medicamentos, infecciones respiratorias activas, enfermedad pulmonar crónica y alteraciones de la función hepática y renal. Por lo tanto, se debe insistir en medidas que permitan un sueño espontáneo, como son un tiempo largo de vigilia previo y el ambiente tranquilo antes y durante el examen. La sedación debe reservarse para casos individuales con una cantidad excesiva de movimientos, cuando sea indispensable evaluar en sueño o si se requiere un registro prolongado. Para dichos casos, los resultados de este estudio apoyan el uso de hidrato de cloral que, en dosis adecuadas, es de alta y rápida efectividad, sin efectos indeseados, salvo la desventaja relativa de la duración de su acción. En niños pequeños se recomienda la administración rectal, ya que no es dolorosa, se evita el rechazo que presenta la vía oral y es eficaz.

\section{Resumen}

Se comparó la efectividad de hidrato de cloral y midazolam como inductores de sueño para electroencefalograma en 59 niños a los que se suministró -previa asignación al azar-, por vía rectal, hidrato de cloral (50 mg $-\mathrm{kg}$ ) (n: 32) o midazolam (1 mg • kg) (n: 27) y 33 niños de la misma edad y condición que durmieron espontáneamente y no requirieron sedación para realjzar los registros. Se logró sueño en todos los niños tratados con hidrato de cloral ante $66,6 \%$ 
de los que recibieron midazolam $(p<0,01)$. La latencia hasta el sueño fue $21,8 \pm 17,5 \mathrm{~min}$ con hidrato de cloral y $117,5+47,2$ min con midazolam $(p<0,01)$. No se observaron influencias de edad, sexo, estado del desarrollo psicomotor, tiempo de vigitia previo o intervalo desde la alimentación hasta la sedación. Estos resultados apoyan el uso de hidrato de cloral por vía rectal en niños pequeños que requieren sedación para EEG.

(Palabras clave: electroencefalografía, inducción de sueño, hidrato de cloral, midazolam.)

\section{Agradecimientos}

Al Dr. Enesto Mellibosky por su colaboracion en el informe de los trazidos. A las Srtas. Lis Muñoz y Verónica Morales por su cuidadoso trabajo de registro de los EEG. A Laboratorios Roche por la donación de midazolam.

\section{Referencias}

1. Strain J, Harvey LA. Foley LC. Campbell JB: Intravenously administered pentobarbital sodium for sedation in pediatric C.T. Radiology 1986: 161: I05108.

2 Thompson J, Schneider 5. Ashwal S, Holder BS, Hinshaw DB. Hasso AN: The choice of sedation for computed tomography in children: a prospective evaluation. Radiology 1982; 143: 475-479.

3. Cook B, Boss J. Nomizu S. Alexunder M: Sedation of children for technical procedures: current standard of practice. Clin Pediatr 1992; $31: 137-142$.

4. Mallol $J$. SIy PD: Effecl of chloral hydrate on arterial oxygen saturation in wheezy infants. Pediatr Pulmonol $1988 ; 5: 96.99$.

5. Harvey SC: Hypnotics and sedatives. En: Goodman Gijman A, Goodman LS, Gilman A. eds. The pharmacological basis of therapeutics, New York: MacMilkan Publishing Co., lnc.. 1990: 339-375
6. Weir MR. Segupeli HH, Tremper LI: Sedation for pedialric procedures. Milit Med 1986; 151: 181-184.

7. Rumm PD, Takao RT, FOx DJ, Atkinson SW: Efficacy of sedation of children with chioral hydrate. South Med J 1990; 83: 1040-1043.

8. Reves JG, Fragen HR. Vinik DJ, Greenblatf $D J$ : Midazolam: pharmacology and uses. Anesthesiology $1985 ; 62: 310-324$.

9. Greenblatt DJ. Sitader RI, Abernethy DR: Current status of benzodiazepines. N Engl J Med 1983; 309 : 410-416.

10. Tolia V, Brennan S, Aravind MK, Kauffman $R$ : Pharmacokinetic and pharmacodynamic study of miduzolam in children during esophagoduodenoscopy. J Pediatr 199!; $119: 467-471$.

II. Sievers TD, Yce iD, Foley ME. Blanding PJ. Berde $C B$ : Midazolam for concious sedation during pediatric oncology procedures: safely and recovery parameters. Pediatrics 1991; 8B: $1172 \cdot 1174$,

12. Diament $M J$, Staniey $P$ : The use of midazolam for sedation of infants and children. AJR 1988; 150: $377-378$.

13. Feld LH, Negus JB, White PF. Oral midazolam preanesthetic medication in pediatric outpatients. Anesthesiology 1990:73:831-834.

14. Starnivoara L. Lindgren L. Klemola VM: Comparison of chloral hydrate and midazolam by mouth as premedicants in children undergoing otolaryngological surgery. Br J Anaesth 1988; 61: 390-396.

15. Saint-Maturice C, Meistelman C, Rey E, Esteve C, de Lauture D, Olive $G$ : The pharmacokinetics of rectal midazolasn for premedication in children. Anesthesiology 1986: 65: 536-538.

16. Speur RM. Yuster $M$, Berkovitz $I$, et al.: Preinduction of anesthesia in children with rectally administered midazolam. Anesthesiology 1991; 74: 670-674.

17. Brown $C R$. Sarnquist FH. Canup CA. Pediey TA: Clinical, electroencephalographic and pharmacokinetic studies of a water soluble benzodiazepine, midazolam maleate. Anesthesjology 1979; 50; 467-470.

1B. Geller E. Niv D. Silbiger A, et al.: RO 15-1788, a benzodiazepine antagonist in the treatment of 34 intoxicated patients. Anesthesiology 1985; 63 [Sup] Aj: 157.

19. Prado de Carvaho $L$, Grecksch $G$, Chapowihier G. Rossier J: Anxiogenic and non-anxiogenic benzodiazepine antagonists. Nature 1983; 301:64-66. 